\title{
NUOVI CONFLITTI \\ E NUOVI SISTEMI PARTITICI ALL'EST. VERSO UN MODELLO ESPLICATIVO
}

\author{
di Pietro Grilli di Cortona
}

I processi di transizione alla democrazia assumono la forma di «ondate» (Huntington 1991) che investono più paesi o aree in differenti momenti di questo secolo. Pur nella varietà delle traiettorie, un aspetto accomuna queste trasformazioni: la costruzione della democrazia significa in primo luogo nascita (o rinascita) dei partiti politici, come principali agenzie di mobilitazione che prendono parte in prima persona ai nuovi processi elettorali, che puntano a gestire direttamente il potere politico e che avranno poi un ruolo determinante nella fase di consolidamento democratico.

Neppure le recenti transizioni est-europee smentiscono questa regola. Anche qui formazioni partitiche emergono già al primo annunciarsi della democratizzazione, con il fine di prepararsi ai primi appuntamenti elettorali e agli impegni di governo che seguiranno. L'obiettivo di questo saggio è individuare e spiegare le prime dimensioni di variazione tra i nuovi sistemi partitici nati in Europa orientale, avendo presente una delle domande cruciali alla base di tanti studi sull'argomento: cosa determina la fisionomia dei sistemi partitici?

Nel delineare preliminarmente alcune peculiarità dei nuovi sistemi partitici est-europei, le precedenti esperienze di democratizzazione in America latina e in Sud Europa costituiranno un occasionale punto di riferimento comparato. I paesi qui considerati per il periodo 1989-1993 sono soprattutto Bulgaria, Cecoslovacchia, Polonia, Romania e Ungheria. Tralasceremo, quindi, sia quelli la cui transizione è determinata in misura preponderante dall'evoluzione dei rapporti con un potente vicino (Germania orientale, Stati dell'ex URSS), sia quelli eredi dello smembramento della Jugoslavia (dove la situazione, peraltro, è ancora in ebollizione), sia infine l'Albania che, rispetto agli altri casi, presenta un leggero sfasamento di tempi essendo la transi- 
zione iniziata con circa un anno di ritardo. Richiami anche a queste esperienze potranno comunque risultare volta a volta utili e interessanti.

Famiglie partitiche emergenti e mancata stabilizzazione dei sistemi partitici

Nel corso del periodo esaminato gli elettori dei cinque paesi sono stati chiamati alle urne più di una volta. In Bulgaria, Cecoslovacchia e Romania si tengono due elezioni parlamentari; in Polonia, tra il 1989 e il 1993, si svolgono tre elezioni legislative; l'Ungheria dopo avere votato nel 1990 per il rinnovo dell'Assemblea Nazionale torna alle urne nel maggio 1994. Ovunque, inoltre, si è tenuta almeno una consultazione per il rinnovo delle amministrazioni locali. Referendum si tengono nella stessa Ungheria (due) e in Romania (uno, boicottato dalle opposizioni). Le elezioni dirette del capo dello Stato sanciscono infine la scelta semi-presidenziale di Polonia e Romania, rispetto a quella sostanzialmente parlamentare degli altri tre paesi (tab. 1). Il periodo, dunque, è breve ma denso di sviluppi.

Per avere un primo quadro dei sistemi partitici est-europei, si possono considerare tre dimensioni principali: 1) le famiglie partitiche emerse con le prime elezioni post-comuniste, 2) l'evoluzione successiva e 3) il tipo di sistemi partitici che vanno formandosi. Sul primo punto, si possono fare due considerazioni. Da un lato, la rinascita dei sistemi partitici sembra avvenire all'insegna di una emulazione degli scenari politici euro-occidentali: prevale ovunque la tradizionale distinzione sinistra-destra; inoltre, le principali famiglie partitiche emergenti sono le stesse che hanno finora predominato nelle assemblee parlamentari dei paesi dell'Europa occidentale e nello stesso Parlamento Europeo: la socialista, la cristiano-democratica e la liberale (tab. 2).

Dall'altro lato, però, una particolarità è visibile nella formazione e nel ruolo dei grandi «cartelli» o «organizzazioni ombrello» che in molti paesi aggregano più partiti, movimenti e orientamenti, uniti nell'opposizione al vecchio regime e nella riaffermazione del valore dell'indipendenza nazionale. Abbiamo così l'Unione delle Forze Democratiche in Bulgaria, il Foro Civico in Boemia e Moravia, il Pubblico contro la violenza in Slovacchia, i Fronti popolari in Estonia e in Lettonia, il Sajudis in 
TAB. 1. Riepilogo delle consultazioni elettorali in Europa orientale (1989-93)

\begin{tabular}{|c|c|c|c|c|}
\hline & $\begin{array}{l}\text { parla- } \\
\text { mentari }\end{array}$ & $\begin{array}{l}\text { presiden- } \\
\text { ziali }\end{array}$ & locali & $\begin{array}{l}\text { referen- } \\
\text { dum }\end{array}$ \\
\hline BULGARIA & $\begin{array}{l}10 \text { e } 17 . \text { VI. } 90 \\
13 . X .91\end{array}$ & gen. 1992 & 13.X.91 & \\
\hline $\begin{array}{l}\text { CECOSLO- } \\
\text { VACCHIA }\end{array}$ & $\begin{array}{l}\text { 8-9.VI.90 } \\
\text { 5-6.VI.92 }\end{array}$ & & 23-24.XI.90 & \\
\hline POLONIA & $\begin{array}{l}4 \text { e } 18 . \text { VI. } 89^{\star} \\
\text { 27.X.91 } \\
\text { 19.IX.93 }\end{array}$ & $\begin{array}{l}25 . X I \text { e } 9 . \\
\text { XII.90 }\end{array}$ & 27.V.90 & \\
\hline ROMANIA & $\begin{array}{l}\text { 20.V. } 90^{\star \star} \\
\text { 27.IX.92 }\end{array}$ & $\begin{array}{l}\text { 20.V.90 } \\
\text { 27.IX.92 }\end{array}$ & $9 . I I .92$ & $\begin{array}{l}\text { dic. } 91 \\
\text { (su nuova } \\
\text { Costit.) } \\
\star \star \star \\
\end{array}$ \\
\hline UNGHERIA & $\begin{array}{l}25 . \text { III e } 8 . \\
\text { IV. } 90\end{array}$ & & $\begin{array}{l}\text { 30.IX e } \\
14 . X .90\end{array}$ & $\begin{array}{l}\text { nov. } 89 \text { e } \\
\text { lug. } 90 \\
\text { (su elez. } \\
\text { C.d.S.) }\end{array}$ \\
\hline
\end{tabular}

Gli organismi legislativi elettivi sono a struttura monocamerale in Bulgaria e Ungheria e bicamerale negli altri tre paesi. Anche in Bulgaria il presidente della Repubblica è eletto direttamente ma non dispone di poteri tali da configurare un potere presidenziale.

* Solo semi-libere, in quanto vengono messi in competizione il $35 \%$ dei seggi del Sejm (camera bassa) e tutti i seggi del Senato.

** Condotte in un clima di intimidazione e di dubbio rispetto per le pari libertà e opportunità per tutte le forze politiche.

$\star \star \star$ Boicottato dalle opposizioni.

Lituania, Solidarnošč in Polonia, la Convenzione Democratica in Romania. Al di là delle differenze ideologiche interne, le esperienze formative alle spalle di queste organizzazioni sono spesso diverse e possono essere distinte sulla base di due criteri: le radici operaie e popolari ovvero elitarie e intellettuali, e l'esistenza o meno di una precedente esperienza più o meno clandestina di opposizione al vecchio regime. Sulla base di questi criteri, Solidarnošč (radici operaie e tradizioni di clandestinità) rappresenta un caso diverso dal Foro Civico (radici più intellettuali e minore ruolo oppositorio nel vecchio regime). Inoltre, data la natura essenzialmente pacifica di queste transizioni (a parte Romania e Jugoslavia), tali coalizioni mancano del tutto delle appendici militari che hanno invece caratterizzato certi fronti o 
ТАB. 2. Seggi delle principali famiglie partitiche in Europa orientale (elezioni 1989-1992)

$\begin{array}{llll}\text { BULGARIA } & \begin{array}{c}\text { CECOSLO- POLONIA ROMANIA UNGHERIA } \\ \text { VACCHIA }\end{array}\end{array}$

Democri-

stiani

$\begin{array}{llll}27 & (40) & 89 & (23)\end{array}$

21

Ex comu-

nisti

$106(211)$

$57(47)$

$60(173)$

$173 \quad(263)$

33

Socialde-

mocratici

Liberal-

democratici

97

(2)

143

- (29)

113

Coaliz. de-

mocratiche

$110(144)$

(168)

(161)

117 (263)

Agrari

$-\quad(16)$

76 (76)

$21-$

44

Naziona-

listi

$24 \quad(23)$

$98 \quad(43)$

$58-$

$73 \quad(38)$

Note: tra parentesi i seggi ottenuti nelle elezioni precedenti; per Polonia e Romania si considerano solo le camere basse. Questo il totale dei seggi delle assemblee parlamentari considerate: Bulgaria 240 (400 alle elezioni precedenti); Cecoslovacchia 300; Polonia 460; Romania 341 (387 alle elezioni precedenti); Ungheria 386.

\section{Avvertenze:}

Cecoslovacchia: nel 1992, tra i democristiani andrebbe contata anche la piccola pattuglia di parlamentari democristiani nella coalizione tra il Partito democratico civico e il Partito democristiano che ottengono, insieme, 85 seggi (che qui consideriamo nella «famiglia» liberale). Tra i liberali nel 1990 dovrebbero essere inclusi parte dei 168 seggi del Foro Civico/Pubblico contro la Violenza.

Polonia: nelle elezioni del 1989 Solidarnošč è ancora unito, e molti suoi parlamentari dovrebbero essere classificati come «democristiani», «liberali» $o$ altro.

Romania: alle elezioni del 1992, fra gli ex comunisti, si conteggiano sia il FSN (43 seggi), sia il FDSN (117 seggi), sia $\mathrm{i}$ «duri» del Partito socialista del lavoro (13 seggi). Nel 1992 i partiti agrari (nazionalcontadini e demo-agrari) entrano nella Convenzione Democratica, pertanto non risultano nella tabella.

comitati di liberazione in altre esperienze di ridemocratizzazione (per esempio Italia e Francia negli anni quaranta).

L'esistenza delle «organizzazioni-ombrello», costituendo un elemento di provvisorietà connesso alla transizione, fornisce già una prima risposta alla seconda questione: se i caratteri del nuovo sistema partitico emersi dalle prime elezioni tendono a fissarsi e a stabilizzarsi o se invece esso è soggetto ad ulteriori trasformazioni. Il che equivale a chiedersi se, sia pure con rife- 
rimento ad un periodo così limitato, esistano i segni di un primo «congelamento» del sistema partitico scaturito dalle prime elezioni, come si verificò in Italia dopo il 1948 e in Giappone dopo il 1952, o se invece la democratizzazione est-europea sia più assimilabile ai casi di Portogallo, Grecia e Spagna degli anni settanta, dove il sistema partitico, dopo le prime elezioni post-autoritarie, ha subito ulteriori trasformazioni in termini sia di redistribuzioni di voti, sia di rotazione al potere (O'Donnell e Schmitter 1986).

E evidente che, sotto questo profilo, il modello verso il quale si muove l'Europa orientale è più il secondo che quello di Italia e Giappone. A conferma di ciò, sottolineo tre sviluppi: a) la frantumazione delle «organizzazioni ombrello», $b$ ) lo sviluppo dei partiti nazionalisti, $c$ ) il manifestarsi di una certa tendenza alla rotazione al potere anche come effetto di mutamenti nella forza elettorale.

Relativamente al punto $a$ ), due casi clamorosi sono quelli polacco e cecoslovacco. Le divisioni all'interno di Solidarno $\check{s}$, già evidenti alle elezioni del 1989, sono confermate con la spaccatura tra Walesa e Mazowiecki alle elezioni presidenziali del novembre 1990 e nel parlamento uscito dalle elezioni politiche del 1991 almeno sette partiti si proclameranno eredi del vecchio sindacato ${ }^{1}$.

Un processo analogo si ha anche in Cecoslovacchia. Nell'Assemblea Federale uscita dalle elezioni del giugno 1990 il Foro Civico (FC) e il Pubblico contro la violenza (PCV) dispongono di 170 seggi su 300 . Un anno più tardi (prima, quindi, delle elezioni legislative del 1992), il gruppo parlamentare del FC si frantuma in cinque nuovi gruppi e quello del PCV si spacca per la scissione del nuovo Movimento per una Slovacchia Democratica (Meciar). Sommando tutti i cambiamenti (si scindono anche i comunisti e l'Associazione per Moravia e Slesia), si passa da un parlamento con sei gruppi, il più consistente dei quali dispone di 170 seggi, a uno con oltre 15 gruppi, il maggiore dei quali dispone di 43 seggi. Neanche in Bulgaria e Romania mancano spinte verso una maggiore articolazione del sistema partitico: si divide il Fronte di Salvezza Nazionale ro-

${ }^{1}$ Unione Democratica, Azione Cattolica, Alleanza dei Cittadini di Centro, Congresso Liberaldemocratico, Alleanza Contadina, Solidarnošc Lavoro, Sindacato Solidarnořc. 
meno (FSN), nasce il Fronte Democratico di Salvezza Nazionale (FDSN, nuovo partito di maggioranza relativa) e si accentuano le differenziazioni interne all'Unione delle Forze Democratiche (UFD) bulgara alla quale tuttavia aderiscono nuovi gruppi.

Complessivamente, accanto a partiti che non hanno fortuna (quelli agrari) o che rifanno capolino negli scenari nazionali (socialdemocratici in Cecoslovacchia), la nascita dei partiti nazionalisti costituisce la novità più rilevante, della quale cercherò più avanti di individuare alcune cause; qui mi limito a tre considerazioni. Anzitutto il diffondersi del nazionalismo mette in crisi la dimensione sinistra-destra: esso, infatti, non si sviluppa solo sulla destra degli schieramenti politici, ma alimenta e influenza anche formazioni di sinistra e contribuisce ad aggregazioni malamente classificabili. La frattura etnica provoca, per esempio, la spaccatura dei comunisti cecoslovacchi (nel Blocco di Sinistra ceco e nel Partito della Sinistra democratica slovacco) e la conversione ultra-nazionalistica dei comunisti serbi; il partito di Meciar in Slovacchia, inoltre, è difficilmente collocabile lungo la dimensione sinistra-destra.

In secondo luogo, la crescita del nazionalismo è un fenomeno che va in direzione opposta allo sviluppo partitico dell'Europa occidentale, a conferma delle forti differenze tra le due Europe (Dogan 1992). Una terza considerazione riguarda poi le differenze all'interno del variegato fenomeno nazionalistico. In tal senso merita forse distinguere tra partiti e movimenti sorti in difesa di specifiche minoranze e partiti e movimenti che hanno invece l'obiettivo di rafforzare il potere di una nazionalità già maggioritaria a danno di altre più piccole. Al primo gruppo appartengono la Minoranza tedesca in Polonia, il Movimento turco in Bulgaria (Movimento per i Diritti e le Libertà, MDL), quello democristiano ungherese in Slovacchia, la Federazione Democratica ungherese di Romania; nel secondo rientrano invece, almeno per alcuni aspetti, il Partito dell'Unità Nazionale romena e la Confederazione per una Polonia indipendente. Non mancano poi ambivalenze e contraddizioni tipiche: come quelle di una frangia del nazionalismo slovacco che rivendicava autonomia da Praga, senza essere però disposta a concederla a sua volta alla minoranza ungherese interna.

Una rotazione al potere (punto $c$ ) si realizza in Bulgaria, con la vittoria di misura della coalizione democratica (1991) che manda all'opposizione gli ex comunisti (ora socialisti), i quali però torneranno al governo l'anno dopo in coalizione 
con l'MDL, e diviene un'aspettativa credibile in Romania, dove il FDSN perde nel 1992 la maggioranza assoluta dei seggi (pur conservando la maggioranza relativa) e molte importanti amministrazioni locali (tra cui Bucarest, Brașov e Timiș oara). In Ungheria il trend negativo per $\mathrm{i}$ partiti governativi inizia con le elezioni locali pochi mesi dopo le prime legislative ed è confermato da vari sondaggi ${ }^{2}$ compiuti successivamente, che danno unanimemente in declino il Forum democratico, $\mathrm{i}$ piccoli proprietari e $\mathrm{i}$ cristianodemocratici e in netta ascesa l'Alleanza dei Giovani Democratici e l'intera opposizione liberaldemocratica, anche se poi gli ex comunisti vinceranno a sorpresa le elezioni del maggio 1994.

In Polonia, una prima rotazione si compie con l'esclusione degli ex comunisti dal governo fin dagli inizi del 1991, una seconda nel 1993 con il ritorno degli stessi al governo dopo aver vinto le elezioni del settembre: quest'ultimo risultato ripete peraltro l'esito delle elezioni lituane dell'anno prima, nelle quali gli ex comunisti di Brazauskas erano tornati al potere sconfiggendo il Sajudis di Landsbergis che aveva gestito la transizione alla democrazia e all'indipendenza. Più difficile dare valutazioni sulla Cecoslovacchia, dove la divisione tra i due stati, a partire dal 1993, ha azzerato la situazione. Ogni commento va pertanto rimandato alle prime elezioni post-scissione.

Il quadro di instabilità è più visibile in Polonia e Cecoslovacchia. Alle elezioni polacche del 1991 troviamo appena due dei partiti di due anni prima: anche gli ex comunisti, infatti, si sono rinnovati nell'Alleanza della Sinistra democratica. A quelle di due anni dopo il quadro partitico appare drasticamente ridotto in seguito all'adozione di un sistema elettorale assai più selettivo, caratterizzato da clausole di esclusione del 5 e dell' $8 \%$, rispettivamente per partiti e coalizioni (Wawrzyniak 1994). In Cecoslovacchia nel 1992 i partiti superstiti sono quattro (due democristiani, il Partito Nazionale slovacco e quello della minoranza ungherese) e otto, invece, i nuovi gruppi parlamentari, tra i quali spicca una formazione di estrema destra, il Partito Repubblicano. In Romania si hanno due spinte opposte: da un lato il FSN si divide, dall'altro la Convenzione Democratica riesce, alle elezioni del '92, ad aggregare partiti che alle elezioni precedenti avevano corso da soli (i nazionalcontadini e gli

2 Sui dati relativi (e sui loro limiti) cfr. Pataki (1991). 
ecologisti). Anche in Bulgaria, infine, l'UFD manifesta una maggiore capacità aggregativa (e con più successo dell'opposizione romena), mentre l'MDL turco si conferma l'ago della bilancia tra le due forze principali.

Per quanto riguarda i sistemi partitici (Sartori 1976) dunque, dal punto di vista numerico predomina un'oscillazione tra il pluripartitismo limitato e l'estremo, con l'unica eccezione dell'Albania (caso, peraltro, qui non preso in esame) che potrebbe stabilizzarsi su un formato bipartitico. Sotto il profilo funzionale è prematuro fare ipotesi e previsioni. La Polonia potrebbe confermarsi come un sistema di pluripartitismo polarizzato (o quasi), malgrado la semplificazione imposta dal nuovo sistema elettorale del 1993. Per gli altri paesi occorrerà aspettare gli effetti della scissione ceco-slovacca, dei nuovi allineamenti partitici che si determineranno in seguito alla disgregazione delle «organizzazioni-ombrello» bulgare e romene, nonché naturalmente le conseguenze di lungo periodo delle leggi elettorali. Un'incognita è poi costituita dall'entità delle spinte polarizzanti prodotte dall'emergere dei partiti nazionalisti.

\section{Le differenze}

Fin qui si sono viste le uniformità più evidenti dei nuovi sistemi partitici est-europei: stesse famiglie partitiche, ruolo delle «organizzazioni-ombrello», presenza dei partiti ex comunisti, tendenza al pluripartitismo limitato od estremo, assenza di stabilizzazione dopo le prime elezioni. In parte, tali caratteri vanno attribuiti alla comune esperienza non democratica, alla contemporaneità delle transizioni, alle cause comuni che le hanno determinate. Tuttavia, alcune prime considerazioni possono essere fatte anche guardando alle varietà dei sistemi partitici emergenti nell'area, tenendo presente che le differenze sono destinate probabilmente ad accentuarsi sotto la spinta delle diverse variabili endogene. Ecco un primo ventaglio di tali differenze:

1) gli ex comunisti perdono il potere in Polonia e in Ungheria (dove però lo riconquistano democraticamente nel 1993 e nel 1994) e in Cecoslovacchia, ma lo conservano in Romania, Bulgaria (con l'interruzione di un anno) e anche in Serbia e Montenegro;

2) le «organizzazioni-ombrello» si formano ovunque meno 
che in Ungheria; inoltre, la sopravvivenza di queste strutture coalizionali ha tempi e durata diversi;

3) i partiti nazionalisti si sviluppano più in certe aree e meno in altre: per esempio, più in Slovacchia che in Boemia e Moravia, più in Romania e Bulgaria che in Ungheria;

4) i partiti confessionali, democristiani o cristiano-sociali, confermando le tradizioni passate, vanno meglio nei paesi a prevalente religione cattolica o protestante che in quelli a maggioranza ortodossa;

5) dai punti suddetti si capisce anche che l'evoluzione dei sistemi partitici tra le prime elezioni post-comuniste e le successive segue nei vari paesi tempi ed itinerari diversi.

Nel tentativo di spiegare queste differenze, considero due variabili principali. La prima, muovendo dall'analisi di Rokkan (Lipset e Rokkan 1967; Rokkan 1982), include l'articolazione e costellazione di quei conflitti e fratture che influenzano la genesi dei sistemi partitici. La seconda fa riferimento, invece, al tipo di transizione, ipotizzando che anche le diverse modalità di questa e le diverse scelte operate abbiano avuto una qualche influenza al riguardo. Queste variabili non esauriscono il complesso e intricato insieme di condizioni che concorrono a spiegare nascita ed evoluzione dei nuovi sistemi partitici. Per esempio, vanno almeno menzionate le influenze dei partiti occidentali, sia in termini di una loro emulazione da parte dei nuovi attori collettivi dell'Est, sia in termini di supporto (organizzativo, finanziario) da parte dei primi atto a favorire la nascita di partiti fratelli in Europa orientale, talvolta nel quadro del rafforzamento della figura del partito pan-europeo. Allo stesso modo, nella fase genetica non è certo mancato qualche serio sforzo di rifarsi a esperienze ideali e organizzative pre-comuniste o addirittura pre-belliche.

Queste spinte, tuttavia, non configurano fattori decisivi di spiegazione. L'emulazione dell'Ovest ha probabilmente operato in una prima fase; ma poi (ad eccezione della Germania Orientale) hanno finito per prevalere spinte interne. In assenza di queste, infatti, partiti formatisi su imitazione di analoghi gruppi occidentali non hanno retto alle prime prove elettorali: penso in particolare all'iniziale pullulare di partiti ecologisti, il cui scarso successo si deve al fatto che i problemi da essi posti non sono ancora percepiti come rilevanti.

Quanto ai tentativi di riesumare forme partitiche del passato, per ora essi non hanno avuto molto successo. I fallimenti o 
gli insoddisfacenti risultati ottenuti da partiti storici, come i socialdemocratici, gli agrari e contadini, i nazional-liberali romeni, configurano un quadro di forte discontinuità, le cui uniche eccezioni sono gli ex comunisti (per i quali, però, forse non è sempre lecito parlare di continuità, e che comunque costituiscono un caso a parte) e qualche singolo partito come i piccoli proprietari ungheresi. Le ragioni di questa prevalente discontinuità con il passato pre-comunista sono state in parte già discusse (von Beyme 1992; Cotta 1992a). In breve, esse richiamano 1) la fragilità e intermittenza delle precedenti esperienze democratiche e partitiche (con la parziale eccezione di Cecoslovacchia e Germania Orientale), 2) la lunga durata della fase non democratica (che non include solo il quarantennio comunista: in Polonia, per esempio, per trovare elezioni libere, o quasi, bisogna risalire al 1930), 3) la caratterizzazione fortemente distruttiva verso la precedente società civile della fase totalitaria comunista.

\section{Tra vecchie e nuove fratture}

Un primo passo è dunque quello di individuare quelle fratture che, combinandosi, danno origine ai nuovi sistemi partitici. Si deve anzitutto verificare il grado di scostamento e l'evoluzione rispetto al modello delle quattro fratture fondamentali (stato/Chiesa, centro/periferia, città/campagna e di classe), considerate come il prodotto di due grandi processi rivoluzionari di mutamento: la Rivoluzione Nazionale avviata in Francia e quella Industriale originata in Inghilterra (Lipset e Rokkan 1967, 14-15); poi quali nuovi conflitti e fratture scaturiscono dagli eventi che segnano il ritorno dell'Est alla democrazia.

Più di quella occidentale, l'Europa orientale costituisce un insieme intrecciato e multiforme di esperienze storiche, di tradizioni culturali e di gruppi etnici e nazionali. Una distinzione di massima ormai accreditata dalla storiografia (Caccamo 1991) è quella tra Europa centro-orientale, caratterizzata dalla tensione Riforma-Controriforma, influenze del mondo tedesco (con fasi alterne di incontro e di scontro), sviluppo di un'economia agricola rivolta verso i mercati occidentali, ed Europa sud-orientale, cioè la regione dei Balcani e dei Carpazi, di religione ortodossa, sotto il secolare influsso dell'Islam, soggetta alle concorrenti influenze degli imperi austriaco e russo e solo in tempi più recen- 
ti (fine secolo XIX-inizi XX) aperta alla penetrazione culturale ed economica dell'Europa. Su questa distinzione fondamentale, che non deve peraltro ignorare aspetti comuni (quale ad esempio le origini recenti dello Stato nazionale in quasi tutta l'area), si sovrappone la differenza tra aree che hanno vissuto una qualche esperienza democratica e di economia di mercato (Germania e Cecoslovacchia) e aree dove invece tale duplice esperienza è ridotta al minimo.

L'innesto del modello comunista (e la sua durata) su questa realtà multiforme ha avuto un effetto omologatore che ha lasciato due tracce principali. La prima è la presenza di partiti ex comunisti che conferisce una forte omogeneità alla sinistra degli schieramenti partitici: resta solo da spiegare (e su ciò torneremo) perché tale presenza è più significativa in certi stati e meno in altri.

La seconda eredità è la fine della frattura di classe tra datori di lavoro e ceti operai. Forte nei territori cechi e in Germania orientale, più debole ( $\mathrm{ma}$ in crescita) negli altri paesi, tale frattura è stata annullata dalle politiche di collettivizzazione e pianificazione intraprese alla fine degli anni quaranta. A tale circostanza va probabilmente attribuito il mancato decollo dei socialdemocratici ${ }^{3}$, una forza ben radicata in gran parte dell'Europa orientale tra le due guerre e il cui tradizionale spazio politico-elettorale è oltretutto già occupato dall'ingombrante presenza dei rinnovati e ben organizzati partiti ex comunisti. In sintesi: se la frattura di classe ha rappresentato, come ci ha indicato Rokkan, un fattore di omogeneizzazione in Europa occidentale, tale da produrre influenze analoghe sui sistemi partitici eurooccidentali, così il suo declino e la sua assenza costituiscono un fattore di omogeneità nei sistemi partitici dell'Europa centroorientale, nei quali poi le differenze sono il prodotto dell'insorgere di nuove linee di frattura e delle loro diverse modalità di presentazione e di espressione.

A queste due eredità dobbiamo poi sommare quelle, meno chiare e dagli effetti meno prevedibili, della quarantennale ope-

${ }^{3}$ Agli inizi della democratizzazione c'è un fermento di gruppi e partiti che si rifanno a un tale orientamento: alla fine del 1989 sono segnalati quattro partiti «socialdemocratici» in Cecoslovacchia, due in Germania Orientale, Bulgaria e Polonia, uno in Estonia, Lettonia, Lituania, Romania e Ungheria. Pochi però ottengono una rappresentanza parlamentare. Tra questi i socialdemocratici tedesco-orientali (78 seggi), quelli romeni (5), quelli bulgari (1) e quelli lituani (8 seggi alle elezioni del 1992). 
ra di riduzione e contenimento degli altri conflitti individuati da Rokkan. In generale, infatti, l'interruzione o l'incapsulamento dei processi di mobilitazione, politicizzazione e democratizzazione operata dai regimi comunisti ha avuto anche l'effetto di ridurre portata e articolazione organizzativa delle vecchie fratture. Più in particolare, sul punto nascono una serie di interrogativi: tale contenimento, che ha significato la distruzione di ogni stabile sistema di articolazione degli interessi, nonché la neutralizzazione delle classi medie e di ogni iniziativa dal basso, quali esiti ha prodotto? L'esaurimento e la scomparsa delle fratture, una loro riproposizione incanalabile nella nuova democrazia, o una loro esplosione incontrollabile conseguente al lungo periodo di artificiale compressione? In altre parole: l'esito è stato solo un indebolimento delle espressioni strutturali e organizzative delle fratture (chiese, partiti, sindacati, gruppi locali), oppure anche uno sbiadimento o addirittura la cancellazione di valori, credenze, identità, autoconsapevolezza, cioè di quegli aspetti culturali che, insieme a quelli strutturali e organizzativi, sono parte integrante delle fratture? Infine: quali nuovi conflitti sono nati?

Un modo per rispondere a queste domande può essere quello di individuare e valutare il peso dei conflitti più significativi presenti o nati nel corso della transizione. Ne emergono tre principali: tra innovatori e conservatori, tra «libertari» promercato e «autoritari» anti-mercato e l'insieme dei conflitti su base etno-territoriale. Inizialmente, la frattura tominante è quella tra innovatori e fautori della democrazia, da una parte, e conservatori e riformatori «tiepidi», dall'altra: ciò risulta sia dalla contrapposizione tra i due gruppi nelle «tavole rotonde», sia dalla formazione delle «organizzazioni-ombrello» in funzione anti-comunista. Tale frattura, in fondo, non fa altro che riproporre sul piano partitico quella tra «i due blocchi distinti di contendenti» che nell'ultima fase dei regimi comunisti e nel corso della transizione ( $\mathrm{ma}$, in almeno tre casi, anche in epoche precedenti: Grilli di Cortona 1989) hanno alimentato il conflitto crescente sul controllo dello stato (Tilly 1993, 323).

Questo conflitto, che raggiunge probabilmente la sua massima espressione nelle prime elezioni libere, specie se queste accentuano il loro carattere plebiscitario (pro o contro il vecchio regime), nell'immediato va incontro a due destini alternativi: può esaurirsi in tempi relativamente brevi, oppure stabilizzarsi e perpetuarsi. Nel primo caso (Ungheria, Cecoslovacchia e Po- 
lonia) la rottura col passato viene confermata, i conservatori hanno la peggio, diventano minoritari o si integrano: la scelta democratica appare comunque irreversibile e la frattura innovatori/conservatori viene gradualmente sostituita dagli altri conflitti. In sintesi, accanto al debole riaffiorare di fratture tradizionali (religiosa, città/campagna) - e combinandosi con esse - acquistano sempre più forza e consistenza gli altri due tipi di contrapposizione. Nel secondo caso invece (Bulgaria, Romania), il conflitto non si esaurisce a causa della forza persistente dei conservatori ex comunisti, pari o addirittura maggiore di quella degli innovatori. Pertanto le altre fratture si cumulano e sovrappongono al primo conflitto, ma non lo sostituiscono.

La formazione dei due schieramenti libertari pro-mercato e autoritari anti-mercato (Kitschelt 1992) è spiegabile con alcune considerazioni. Qual è, infatti, l'impatto del cambiamento sui ceti ereditati dal vecchio regime? Il ritorno del mercato offre nuove opportunità (di miglioramento del proprio status, di arricchimento), ma porta con sé anche nuovi problemi. E la disoccupazione tra gli operai delle non competitive imprese statali, nonché il peso della concorrenza interna ed esterna su contadini e imprese rurali, spingono questi ceti a chiedere ovunque il rinvio della chiusura delle aziende non produttive, sussidi, tariffe di favore, misure protezionistiche (Jasiewicz 1992) - e comunque a manifestare diffidenza verso la transizione al sistema di mercato.

Da tutto questo emergono alcuni punti che conviene riassumere. Anzitutto, lo sviluppo dello schieramento pro-mercato (liberaldemocratici, moderati, in parte cristiano-democratici) ha origine e si rafforza nella precedente opposizione al regime comunista, a cui si pone in alternativa in nome dei valori occidentali. In secondo luogo, le idee anti-mercato tendono a svilupparsi in un momento successivo. Una volta passata l'euforia iniziale alimentata anche dall'idea che l'introduzione del mercato avrebbe sciolto tutti i nodi economici, emergono i primi gravi problemi: 1) il mercato (specialmente nella primissima fase di rinascita incontrollata) mette in evidenza anche tutti i suoi lati più inumani; 2) sorgono le prime discussioni in materia di strategie da seguire e di formulazione e applicazione delle nuove leggi. A quest'ultimo proposito, ecco alcuni dei «dilemmi» più comuni: quali aziende privatizzare e quali mantenere nell'orbita dello stato? Quali incentivi dare ai dipendenti delle aziende da privatizzare per far loro «ingoiare il rospo» della de-statalizza- 
zione? Limitare o no (e come) gli investimenti stranieri? Dalla discussione su questi punti (e di solito a prima legislatura iniziata) prende forma in tutte le sue sfumature la spaccatura tra favorevoli e contrari al mercato. Terzo punto: nello schieramento anti-mercato (ex comunisti, populisti, nazionalisti, tradizionalisti riemergenti) confluiscono quei gruppi sociali che si convincono sempre più di non avere capacità e risorse spendibili o convertibili nell'emergente sistema di mercato e che pertanto avvertono il pericolo di una vera e propria degradazione professionale.

Da ultimo, resta da spiegare perché le forze pro-mercato fanno più fatica ad affermarsi in certi paesi anziché in altri. Sul punto ritengo che la diversa forza dei due schieramenti e i diversi allineamenti partitici che ne derivano dipendono da tre fattori strettamente connessi che alimentano un clima culturale più favorevole al mercato: $a$ ) il livello di industrializzazione e di sviluppo economico (più è stato elevato e più il ritorno al mercato ha probabilità di essere accolto positivamente); $b$ ) l'esistenza o meno di passate tradizioni di economia di mercato, magari a tratti riaffiorate sotto il comunismo (più esse sono forti e più il ritorno al mercato ha probabilità di essere percepito come un processo normale, con difficoltà superabili); c) la gradualità o meno della transizione economica: se e in quale misura riforme decollettivizzanti e liberalizzatrici erano già state intraprese prima della democratizzazione (in caso affermativo il cambiamento è meno traumatico).

Emerge così un primo quadro esplicativo delle differenze. I partiti libertari pro-mercato tendono ad essere più forti in quelle aree che in passato hanno conseguito più alti livelli di industrializzazione e con tradizioni più forti di economia di mercato (Repubblica Ceca e Germania Orientale), oppure dove limitate riforme nel senso del mercato hanno preceduto e preparato la democratizzazione (Ungheria); dove queste condizioni hanno operato meno (Polonia, Bulgaria, Slovacchia, Slovenia, Croazia) si sviluppano più forti partiti collocati nel settore autoritario anti-mercato dello spazio competitivo ${ }^{4}$. $\mathrm{Al}$ polo opposto rispet-

4 Le aree di maggior tradizione industriale, e dove pertanto ha potuto radicarsi un proletariato industriale fin dalla fine del XIX secolo, sono Boemia e Moravia, la Germania orientale, la Polonia occidentale (Lodz e la Slesia) e l'area di Budapest. Più tardi, altri centri industriali di qualche rilievo sono sorti in Slovenia e Croazia (Lubiana, Zagabria, Celje, Maribor), nelle aree dell'industria petrolifera romena e intorno ai grandi centri urbani (Sofia, Belgrado, Bucarest, ecc.). 
to a Repubblica Ceca e. Ungheria, troviamo infine Romania, Albania, Serbia, Macedonia e Montenegro, con una ulteriore accentuazione del versante autoritario anti-mercato e forti difficoltà di affermazione per i partiti del versante opposto'.

La diversa iniziale collocazione dei vari paesi riflette la differente presenza di categorie che dispongono o meno di risorse e capacità riutilizzabili e riciclabili nel nuovo sistema economico. Contano, a questo proposito, sia il livello di istruzione e di specializzazione professionale, sia il tipo di collocazione professionale. In genere, per esempio, nel passaggio ad un'economia di mercato gli operai specializzati hanno più probabilità di conservare la propria funzione e il posto di lavoro (hanno migliori probabilità di ricollocarsi dignitosamente) rispetto a quelli senza specializzazione. Tuttavia, i lavoratori specializzati delle vecchie industrie pesanti si troveranno certamente più in difficoltà di quelli impiegati in industrie leggere più suscettibili di essere rese competitive con minore costo.

Riepilogando, la trasformazione ad un'economia di mercato troverà tanto meno oppositori quanto più 1) l'economia comunista ha subito processi di riadattamento e modernizzazione, 2) è alto il livello di istruzione e specializzazione professionale, 3) ampio e sviluppato risulta, al momento del crollo del regime, il settore economico extra-statale (la «seconda economia»: Hankiss 1990). Queste condizioni consentono di minimizzare gli effetti di quella cultura politica di «egualitarismo autoritario» (Offe 1992), ereditata dal vecchio regime e compatibile solo con il mantenimento di un sistema economico gestito dallo stato.

Ne segue che, in concreto, le categorie che potranno meglio integrarsi sono i manager, i professionisti (medici, giuristi, ingegneri, ecc.), l'intelligencija tecnica e amministrativa, gli intellettuali, gli operai specializzati dell'industria leggera. Maggiori difficoltà d'integrazione avranno invece pensionati, burocrati, lavoratori agricoli, operai non specializzati, personale dei vecchi apparati di sicurezza, dipendenti di aziende da smantellare.

5 Pur integrandolo e modificandolo in alcune parti, sul punto mi ispiro al modello esplicativo di Kitschelt (1992). Tuttavia, quanto qui si vuole spiegare è la forza complessiva dei due poli contrapposti (pro- e anti-mercato), non quella dei singoli partiti all'interno di ciascun polo. Va da sé, inoltre, che quello qui delineato è solo il quadro iniziale. Successive oscillazioni nei rapporti di forza tra gli opposti schieramenti sono (e saranno) per lo più spiegabili con l'impatto dei costi sociali del ritorno del mercato (vedi i recentissimi sviluppi ungheresi). 


\section{L'impatto del nazionalismo}

La destinazione partitica delle categorie che più rischiano di essere danneggiate dall'introduzione del mercato imbocca varie direzioni. Gli ex comunisti sono quelli che più frenano le spinte alla transizione economica, mettendo in guardia dalle conseguenze nefaste in termini di disoccupazione, smantellamento delle strutture di welfare, peggioramento delle condizioni delle categorie più deboli. I partiti agrari, inoltre, cercano (finora con scarso successo) di farsi portavoce degli interessi rurali che temono di pagare costi eccessivi per la ricostruzione e riconversione dell'industria: per la verità, questi partiti esprimono più un generico desiderio di continuità con il passato che la persistenza di una reale frattura città/campagna. Talvolta essi spingono anche per una redistribuzione delle terre coltivabili agli antichi proprietari o ai loro eredi (come i piccoli proprietari in Ungheria). Il declino dei partiti rurali, rispetto al primo dopoguerra, non significa però la totale scomparsa della frattura città/campagna. Questa, piuttosto, tende a fondersi e a sovrapporsi alla frattura tra favorevoli e contrari al mercato: un dato emerso in molti paesi, infatti, è che mentre gli abitanti delle città hanno un atteggiamento generalmente più favorevole verso le trasformazioni economiche e la democratizzazione, dalle campagne provengono le maggiori resistenze che poi, a livello elettorale, si traducono in un appoggio per i partiti contrari a riforme troppo rapide e radicali.

Un destinatario degli elettori anti-mercato sembra essere anche l'eterogenea ma crescente famiglia dei partiti nazionalisti. Il nazionalismo è storicamente radicato nel mosaico etnico dell'Europa centro-orientale (Bowers 1992) e il centralismo comunista ne ha impedito ogni maturazione e sedimentazione da cui scaturisse una qualche forma di modus vivendi o di accomodamento consensuale: il nazionalismo costituisce pertanto anche uno degli effetti del lungo periodo di compressione dei conflitti attuato in precedenza. Sebbene penalizzato nelle sue espressioni organizzative (con la messa al bando di gruppi e movimenti e con il divieto di ogni manifestazione e celebrazione pubblica), esso è stato perpetuato e trasmesso nei suoi valori di base da strutture meno controllabili (per esempio, la famiglia). Se il nazionalismo del secolo scorso ha accompagnato le spinte disgreganti con spinte anche ricostruttive e unificanti (il crollo dell'Impero Asburgico ha significato anche la riunificazione di va- 
rie popolazioni sotto nuove forme statali come l'Italia, la Jugoslavia e la Cecoslovacchia), nel nazionalismo post-comunista, con l'eccezione della riunificazione tedesca, l'ondata secessionista sembra prevalere; al punto che, oltre a Cecoslovacchia e Jugoslavia, ha investito anche $\mathrm{i}$ territori che avevano fatto parte dell'Impero Russo, producendo globalmente uno sminuzzamento di stati spesso reciprocamente ostili.

Alle prime elezioni (1989-90) il richiamo nazionalista appare poco rilevante (nel '90 in Slovacchia nessun partito è apertamente scissionista), mentre assume qualche consistenza dove gli ex comunisti sono ancora forti (Bulgaria, Romania, nazioni eredi della Jugoslavia): qui, infatti, non solo le minoranze nazionali (Turchi in Bulgaria, Ungheresi in Romania, ecc.) colgono subito l'opportunità e competono alle elezioni anche perché percepiscono la minaccia di una ambigua decomunistizzazione, ma gli stessi partiti eredi del passato regime sposano fin dall'inizio la fino ad allora repressa ostilità nazionalista della maggioranza verso le minoranze interne.

$\mathrm{Si}$ aggiunga poi che esiste un legame di continuità-contiguità tra il conflitto pro/anti-mercato e la rinascita del nazionalismo. Non è un caso, infatti, che quest'ultimo germini con più vigore nelle aree dove è più forte l'ostilità contro il mercato, giustificata di solito adducendo argomenti dal chiaro sapore nazionalista: l'esigenza di preservare la propria cultura e le proprie tradizioni, il timore dell'invasione degli investimenti stranieri, la diffidenza verso l'esterno, la paura che la concorrenza internazionale finisca per schiacciare l'economia nazionale, la volontà di conservare le proprie particolarità produttive. Tutto questo concorre a spiegare l'occasionale saldatura (in funzione anti-mercato) tra una destra tradizionalista, centralista, nazionalista e anti-occidentale e una sinistra conservatrice, nostalgica e statalista: una saldatura, peraltro, che concorre ulteriormente a erodere il ruolo esplicativo della dimensione sinistra-destra.

In questa sede il fenomeno del nazionalismo est-europeo interessa soprattutto se e in quanto implica la traduzione in schieramenti partitici dei conflitti etno-territoriali. È dunque importante, in prima istanza, capirne la portata effettiva, le modalità di espressione partitica e le conseguenze a livello istituzionale e statale. Se è vero, infatti, che anche nei paesi dell'Europa occidentale non mancano fratture etniche, religiose o territorial-culturali (Spagna, Belgio, Svizzera, Irlanda), è altrettanto vero che in Europa orientale operano alcune condizioni aggravanti. In 
sintesi, muovendo anche da alcune riflessioni di Mattei Dogan (1993), è utile ricordare i seguenti punti: 1) si tratta di stati giovani con frontiere ancora contestate o comunque percepite come non definitive; 2) predomina un sentimento di reciproca diffidenza tra gli stati (specie se confinanti); 3) non si è sviluppata alcuna forma di interdipendenza o complementarietà economica; 4) tutti gli stati est-europei hanno consistenti minoranze etnico-linguistiche all'interno o componenti allogene che rivendicano forme di autonomia, quando non addirittura il ritorno alla madrepatria; 5) le esperienze nel corso delle quali tali minoranze hanno avuto modo di articolare le proprie domande e rivendicazioni in un contesto democratico, pluralistico e negoziale sono state limitate; 6) i partiti ex comunisti, capovolgendo ogni loro precedente impostazione internazionalista, incoraggiano in vari casi il sentimento e la mobilitazione su base nazionale.

La rilevanza dei conflitti etnici e i loro effetti sul sistema partitico e istituzionale sono influenzati da molti fattori. Tra questi, la dislocazione dei gruppi etnici e nazionali sul territorio, il fatto se la frattura etnica è rafforzata da altre fratture (linguistica, religiosa, urbano-rurale, ecc.), la formazione politico-culturale delle etnie maggioritarie nello stato, la sequenza delle prime consultazioni elettorali (se prima regionali e poi statali-federali, o viceversa). La dislocazione può assumere varie forme (Dogan 1993): a) separazione (come quella tra Cechi e Slovacchi); $b$ ) intreccio, quando le nazionalità si intersecano in aree con confini poco definibili, dai contorni sinuosi e frastagliati (Bosnia e Croazia); c) accerchiamento, quando un'etnìa concentrata e maggioritaria in un territorio è circondata da un'altra etnìa maggioritaria nello stato (gli Ungheresi nel centro della Romania); d) dispersione sul territorio di individui e nuclei familiari di un'etnìa minoritaria (Russi in Ucraina o Slovacchi nella Repubblica Ceca).

Separazione e intreccio, oltre che una forte incidenza sul sistema partitico (duplicazione di partiti, partiti rappresentativi di gruppi etnici), possono produrre effetti importanti a livello statale. Nel primo caso sono più probabili esiti pacifici e consensuali: o un accordo dal quale scaturisca un sistema di convivenza (magari su basi consociative); o una scissione, tanto più probabile quanto più la frattura etnica risulti rafforzata da altri conflitti e da élites ${ }^{6}$ che ne massimizzano la portata. La scissio-

${ }^{6}$ Higley e Burton (1989) pongono in relazione il grado di unità delle élites e la stabilità del nuovo regime democratico. 
ne ceco-slovacca è stata anche lo sbocco di profondi dissensi circa le scelte in ordine alla ricostruzione economica (Blaha e Wehrlé 1992; Deis 1992; Capek e Sazama 1993). Nel caso dell'intreccio, la maggiore difficoltà di pervenire a separazioni territoriali e amministrative rende più probabile l'esplosione di conflitti violenti (come nella ex Jugoslavia). Nelle situazioni di accerchiamento e dispersione le conseguenze restano probabilmente limitate al sistema partitico e, semmai, alla concessione di forme di autonomia amministrativa.

Inoltre, la natura dei conflitti etno-territoriali risulta influenzata dalla circostanza se nell'etnia maggioritaria (per esempio, in una situazione di «accerchiamento») predomina una cultura politica democratica, negoziale e cosmopolita o, al contrario, sostanzialmente autoritaria, dogmatica e particolaristica. $\grave{E}$ chiaro che nel primo caso sono più probabili soluzioni di tipo cecoslovacco, mentre nel secondo i conflitti rischiano di perpetuarsi (Bulgaria e Romania) o di arrivare al caso estremo della guerra civile (come negli stati dell'ex Jugoslavia e in alcuni dell'ex URSS).

Infine, nel corso di una transizione democratica, lo sviluppo di un sistema partitico orientato su basi statali oppure regionali e particolaristiche può essere incoraggiato dalla particolare sequenza delle prime elezioni libere. In altri termini, quando una entità statale include al proprio interno più componenti etniche e nazionali, il fatto che si tengano prima elezioni unitarie generali o elezioni regionali può essere importante ai fini della fisionomia del futuro sistema partitico. Nel primo caso si rafforzano gli incentivi per la nascita di partiti su base statale o federale o comunque sovranazionale, fondati quindi su questioni di carattere generale che prescindono dalle differenze nazionali o regionali; nel secondo, invece, viene incoraggiato lo sviluppo di forze su base, appunto, regionale o nazionale, incoraggiando forme di contestazione anti-statale e anti-unitaria che indeboliscono la legittimità dello stato centrale.

Questo aspetto è esaminato da Linz e Stepan (1992) con riferimento ai casi di Spagna, URSS e Jugoslavia. Mentre la Spagna esordisce con tre consultazioni generali, negli altri due casi le prime elezioni «formative» per il sistema partitico sono quelle a livello di repubblica, con forti incentivi quindi alla differenziazione regionale e all'indebolimento della legittimità statale ${ }^{7}$.

7 In Spagna si tengono due referendum e un'elezione generale (1977). In Jugosla- 
Sia chiaro: la sequenza elettorale non spiega tutto. La scissione ceco-slovacca, malgrado le prime elezioni su base federale, non smentisce necessariamente l'importanza relativa delle sequenze elettorali; pone semmai l'accento sul peso di altre variabili, quali l'irriducibilità di certe fratture etniche, la sovrapposizione di più fratture e, nel caso specifico, la percezione dell'unità cecoslovacca come di un mero artificio storico imposto dai Trattati della Prima guerra mondiale.

Un problema a sé, infine, è costituito dalla diversa incidenza della frattura stato-Chiesa e dal fatto se essa rafforza o meno quella nazionalista. Abbiamo visto che mentre nei paesi dell'Europa centrale e negli stati baltici si sono sviluppati partiti confessionali e a sfondo religioso (sia pure non particolarmente significativi nel panorama generale), il fenomeno non ha la stessa rilevanza in Bulgaria e Romania. Una spiegazione potrebbe fare riferimento al diverso ruolo svolto dalle Chiese nazionali ortodosse rispetto a quelle cattoliche e protestanti. In Bulgaria e Romania è mancato quel conflitto Chiesa-stato altrove alimentato dal crescente interventismo del secondo nel campo dell'istruzione, ed è mancata anche qualsiasi contrapposizione rilevante nel quarantennio comunista. A questo proposito, va anche detto che quanto più le chiese hanno fornito sostegno al regime non democratico, tanto meno probabile appare la rinascita di partiti significativi a sfondo religioso ${ }^{8}$. Le Chiese ortodosse, infatti, sono state più uno strumento dello stato che non il punto di coagulo di un qualche gruppo si opposizione e di dissenso (come in Polonia), perpetuando del resto un modello di relazioni che ha radici lontane: nel Cesaropapismo bizantino, nel sistema dei millet (singole comunità religiose) nei Balcani sotto l'occupazione ottomana e anche nella tradizione di subordinazione allo stato della Chiesa russa ortodossa (Ramet 1988; Papadakis 1988).

Non che, nella stessa area ortodossa, siano mancate eccezioni a questo modello di rapporti, con conflitti tra poteri politici e religiosi e interventi delle Chiese in campo politico e sociale. Tuttavia, la subordinazione della Chiesa allo stato, che in certi casi si è fondata sull'aspirazione ideale ad un'unità di Chiesa e

via non si tengono affatto elezioni federali libere. In URSS si tengono elezioni federali nel 1989, ma ancora largamente non competitive (White 1990).

${ }^{8}$ Tutto sommato, sia pure in contesti molto diversi, i casi di Spagna e Portogallo conferriano l'ipotesi. 
stato in una società cristiana universale, prevale nell'itinerario storico dell'area e contrasta con il dualismo che, con alterne vicende, segna il percorso storico dell'Europa occidentale, dualismo che è in larga parte alla base dello sviluppo di partiti confessionali.

Nel complesso, tuttavia, e al di là di queste differenze, le fratture religiosa e città/campagna, sebbene non scomparse e sempre suscettibili di riattivazione, risultano quelle più indebolite dalla fase comunista. In ambedue i casi, l'eccezione più vistosa è la Polonia, dove infatti la Chiesa ha continuato a svolgere un ruolo sociale attivo anche sotto il comunismo e dove l'agricoltura non è stata interamente collettivizzata: se ne vedono i segni nella presenza di più partiti religiosi e rurali. Quanto ai richiami nazionalistici invece, anziché risultare indeboliti essi sono stati solo compressi ed esasperati, ponendo così le basi della loro riesplosione successiva.

\section{Transizione democratica e sistemi partitici}

Dalle esperienze est-europee non si desume un legame diretto tra la continuità/discontinuità della transizione (Morlino 1986) e una particolare conformazione del sistema partitico. Più specificamente, ferma restando la presenza ovunque di formazioni eredi dei vecchi partiti unici, non c'è una relazione transizione continua/forte partito ex comunista o, viceversa, transizione discontinua/debole partito ex comunista: in Ungheria, il vecchio partito unico esce minoritario dalle prime elezioni pur essendo stato il principale promotore della democratizzazione; in Romania, invece, gli ex comunisti diventano maggioritari malgrado una transizione che assume tutte le apparenze di una rottura rivoluzionaria col comunismo (tab. 3).

Tuttavia, è già emerso come la transizione influisca sulla conformazione del sistema partitico: la particolare transizione della Germania dell'Est ha favorito lo sviluppo di partiti in buona misura simili a quelli della Germania federale; ugualmente, si è visto come la scelta di tenere prima elezioni su base statale o su base regionale possa limitare o incoraggiare lo sviluppo di partiti di orientamento regionale o nazionalistico. In generale, è nella transizione che prende forma un'intelaiatura di condizionamenti, freni e opportunità politiche (Tarrow 1991) che canalizzano la mobilitazione inducendo certi partiti a organizzarsi. 
TAB. 3. Tipo di transizione ed ex comunisti

\begin{tabular}{lll}
\hline & Transizione & Partito ex comunista(*) \\
\hline UNGHERIA & continua & minoritario e all'opposizione \\
POLONIA & continua-mista & minoritario e all'opposizione \\
BULGARIA & mista & maggioritario e al governo \\
CECOSLOVACCHIA & mista & minoritario e all'opposizione \\
ROMANIA & discontinua & maggioritario e al governo \\
\hline
\end{tabular}

(*) Alle prime elezioni libere.

Intanto, la dinamica e le modalità che caratterizzano la transizione hanno un'influenza su presenza, ruolo e durata delle «organizzazioni-ombrello». Infatti, queste sorgono: 1) per contrapporre una forza unitaria all'élite del vecchio regime onde obbligarla ad accelerare i tempi della transizione e condizionarne le varie fasi; 2) perché le prime elezioni libere sono percepite in realtà come un referendum pro e contro il comunismo; 3) perché si è consci che la democratizzazione non è un processo scontato e irreversibile. Sussiste ancora la convinzione che le resistenze al cambiamento sono forti, che gli ex comunisti controllano settori decisivi della società (servizi segreti, parte dell'esercito e della burocrazia), che le trasformazioni del partito unico sono più che altro «gattopardesche», e così via.

Ne deriva che quanto più si ritiene minacciata la democratizzazione, tanto più è probabile che le forze democratiche restino coalizzate in un fronte unico. La persistenza di questo, mettendo in secondo piano le altre fratture, tende a congelare il sistema partitico sul bipolarismo comunismo/anti-comunismo e a impedirne o ritardarne il pieno dispiegamento. Tale situazione è destinata a durare almeno finché gli ex comunisti restano forti o non forniscono garanzie sufficienti di adesione alla nuova democrazia. Un loro indebolimento o una loro piena integrazione provocherà la frantumazione del fronte avverso. Tutto ciò è ben illustrato dai casi contrapposti di Ungheria, da una parte, e Bulgaria e Romania, dall'altra.

In Ungheria i comunisti hanno saputo rinnovarsi e avviare la transizione con decisione cercando di associarvi i neonati partiti di opposizione. Questi, pur rifiutando di andare al go- 
verno e malgrado la loro debolezza, conseguono qualche successo: per esempio, sventando (con una vittoria di misura al referendum del 26 novembre 1989) il progetto comunista di tenere elezioni presidenziali prima di quelle parlamentari che, se realizzato, avrebbe quasi sicuramente significato l'elezione di un esponente del più preparato e organizzato partito al potere. $\mathrm{La}$ vittoria fornisce alle opposizioni due prime certezze: che i comunisti accettano le regole democratiche e che possono essere elettoralmente battuti.

Ben diversi gli scenari bulgaro e romeno. In questi paesi i comunisti $a$ ) non hanno alcun passato di riformatori, $b$ ) avviano e aderiscono alla transizione solo perché spinti dalle circostanze e con dubbi propositi di sincera democratizzazione, $c$ ) sono più forti dei comunisti ungheresi e mostrano un maggiore controllo di tutti i settori chiave della società. Queste circostanze spingono i partiti democratici a dare più importanza a ciò che li unisce (contrapposizione all'ex partito unico) che a ciò che li divide. In generale, dunque, una democratizzazione più difficile e ostacolata quale quella di Bulgaria e Romania - a causa della persistente forza dei comunisti e del peso di condizioni storiche tradizionalmente meno favorevoli ad una evoluzione democratica - si riflette sul sistema partitico rallentandone lo sviluppo. E tale rallentamento assume principalmente la forma di una perpetuazione della linea di frattura originaria della transizione (pro o contro il comunismo), rinviando l'emergere delle altre fratture che alimentano l'articolazione del sistema partitico a quando sarà confermata definitivamente l'opzione democratica. Il ritorno di un «pericolo comunista» può anche interrompere $\mathrm{e}$ bloccare lo sviluppo del sistema partitico, ricreando aggregazioni e coalizioni democratiche come agli inizi della transizione. $\grave{E}$ il caso dell'Ucraina nei primi mesi del 1993, il cui sistema partitico pare quasi aver innestato la retromarcia rispetto all'anno precedente.

Qualche restante considerazione deve essere fatta poi circa il ruolo di altri tre elementi della transizione: 1) le «tavole rotonde», 2) la mobilitazione popolare e 3) i sistemi elettorali adottati. Le «tavole rotonde» sono un momento centrale delle transizioni est-europee anche perché generano le prime decisioni relative a tempi e fasi della costruzione democratica e alla fissazione delle nuove regole. Il ruolo dei partiti, in questa fase, è debole e di solito i patti realizzati nelle «tavole rotonde» sono influenzati dal grado di sincera adesione al rinnovamento da 
parte degli ex comunisti, dalla pressione della piazza, da eventi internazionali. Questi ultimi, per esempio, spiegano i diversi esiti dei negoziati polacco e ungherese: il fatto che l'opposizione magiara ottenga assai più di quella polacca dipende in larga parte dalla circostanza che la Polonia è il primo paese ad aprire la serie di transizioni e l'atteggiamento sovietico costituisce ancora un'incognita (Batt 1991). Le elezioni polacche dell'89, dunque, risentono di questo clima di incertezza: ai comunisti viene lasciata una quota fissa di seggi nel Sejm (il 65\%) e ciò concorre a mantenere ancora per un po' l'unità di Solidarnošč e a ritardare l'evoluzione dei partiti.

Tuttavia, la conformazione del sistema partitico, almeno nella fase iniziale, viene influenzata dal tipo - o meglio, dal contenuto - della trattativa in corso. Un conto è, infatti, se le questioni all'ordine del giorno riguardano se e quanta democrazia introdurre; un altro conto, invece, è se lo scopo della trattativa concerne solo le modalità di avvio della democrazia stessa (sulla cui rinascita nessuno dei partecipanti alla «tavola rotonda» esprime dubbi), la mappa istituzionale e costituzionale del nuovo regime. Nel primo caso (Bulgaria, Polonia, Romania, in parte Cecoslovacchia) è più probabile che le forze non comuniste rimangano inizialmente coalizzate sotto una stessa organizzazione-ombrello; nel secondo (Ungheria), la certezza circa l'imminente introduzione della democrazia e lo svolgimento di elezioni completamente libere induce le varie forze politiche a presentarsi divise e a competere per ottenere il maggior numero possibile di opportunità, risorse e vantaggi per sé.

Il ruolo della piazza, poi, può essere duplice. Da una parte, le manifestazioni di massa sono un potente acceleratore della transizione, in grado di influire notevolmente sullo svolgimento delle «tavole rotonde». Così in Cecoslovacchia e Germania Est (più controversi gli eventi romeni). Dall'altra, si può notare una relazione tra il livello di partecipazione di massa alla transizione e quello successivo di partecipazione elettorale. In altri termini, quanto più la transizione rimane un processo elitario, limitato ai vertici istituzionali e a quelli di partiti con scarso seguito, tanto più è probabile anche una scarsa partecipazione alle prime elezioni post-comuniste. I casi polacco e ungherese ne sarebbero una conferma (Grilli di Cortona 1991a, 307).

Una delle scelte più significative della transizione è poi siamo al terzo elemento - quella dei sistemi elettorali. Non è qui il caso di soffermarsi sulle ragioni di una scelta quasi unani- 
me a favore dei sistemi proporzionali, anche se l'ipotesi avanzata da Rokkan per l'Europa occidentale («una convergenza di pressioni dal basso e dall'alto») potrebbe servire, riadattata, come base esplicativa anche per le nuove realtà est-europee. In altre parole, ex comunisti e opposizioni democratiche prendono il posto, rispettivamente, degli artefici di pressioni convergenti considerati da Rokkan: i vecchi partiti timorosi di perdere tutto e $\mathrm{i}$ ceti operai in ascesa (Lipset e Rokkan 1967, 32). I sistemi elettorali adottati per le camere basse vanno da un massimo di proporzionalità come in Polonia nel 1990, con un sistema che prevede dimensioni mediamente ampie dei collegi ma privo di una vera e propria clausola di esclusione (Webb 1992), al caso opposto dell'Ungheria che ha adottato un sistema misto nel quale, però, gli effetti maggioritari prodotti dal doppio turno in collegi uninominali (che eleggono il $45,6 \%$ dei parlamentari) non sono compensati dal voto su base proporzionale (da cui scaturiscono il $54,4 \%$ dei parlamentari) (Hibbing e Patterson 1992; Körösényi 1990). Tra questi casi polari troviamo poi, in ordine di crescente proporzionalità, Bulgaria, Cecoslovacchia e Romania: la prima con un sistema misto e una clausola di esclusione del $4 \%$; le altre due nazioni con leggi proporzionali attutite da clausole di esclusione rispettivamente del 5 e del $3 \%$. Questo quadro iniziale subisce delle modificazioni, per esempio con l'adozione in Polonia di una legge più restrittiva i cui effetti sono già visibili alle elezioni del settembre 1993.

Tuttavia, la questione è se e quanto i nuovi sistemi elettorali hanno concorso a plasmare i neonati sistemi partitici est-europei $a b$ initio. Sappiamo, in generale, che gli effetti dei primi sui secondi si dispiegano essenzialmente nel lungo periodo: per esempio, contribuendo a mantenere un determinato formato partitico, oppure modificando nel tempo il comportamento degli elettori attraverso la sotto- o sovra-rappresentazione di certi partiti (Fisichella 1993). Si aggiunga poi che l'azione dei sistemi elettorali viene spesso sopravvalutata: che la frammentazione della camera bassa polacca, per esempio, non fosse tutta da addebitare al primo sistema elettorale adottato è dimostrato dal fatto che il Senato, eletto con legge maggioritaria, era quasi altrettanto frammentato (Lijphart 1992).

Fermo restando, dunque, che difficilmente i sistemi elettorali possono esser considerati variabili indipendenti nei confronti dei sistemi partitici, fin qui un loro effetto accertabile è stato quello di filtro: la legge elettorale, infatti, impedisce che 
tutti i partiti che si presentano alle elezioni ottengano una rappresentanza e tale funzione è tanto più cruciale in paesi che hanno appena riacquistato la democrazia e nei quali vi è una esplosione di gruppi e partiti in virtù del principio che tutti vogliono provarci. Più in generale, infatti, guardando anche ad altri casi (Turner 1993, 341-343), sappiamo che le prime elezioni post-autoritarie sono quelle nelle quali si realizza il massimo numero di scelte per gli elettori.

Ma quanti, tra i partiti che ci provano, entrano in parlamento alle prime elezioni? Apparentemente il filtro ha maglie più strette in Romania (7 su 74) e in Bulgaria (3 su 37); un po' meno in Ungheria (7 su 45), in Cecoslovacchia (11 su 43) e in Polonia (18, più 11 partiti che conquistano un seggio ciascuno, su 67) (McGregor 1993). In concreto, però, l'accertamento dell'effetto-filtro non va per ora molto al di là di questi dati quantitativi: solo l'osservazione di una successione di elezioni potrà darci maggiori ragguagli qualitativi (indicandoci, per esempio, fino a che punto il sistema di incentivi e disincentivi messo in atto dal sistema elettorale produrrà quali effetti duraturi). Inoltre, i dati relativi a Bulgaria e Romania risultano falsati dalla presenza persistente dei fronti democratici coalizionali, la cui futura frammentazione porrà fine all'effetto semplificatore fin qui prodotto, modificando in modo forse anche rilevante la fisionomia del sistema partitico.

\section{Considerazioni conclusive: le cause dell'instabilità partitica}

Un obiettivo di questo saggio era spiegare come e perché paesi usciti da una comune quarantennale esperienza di comunismo sviluppino sistemi partitici così diversi già a partire dalle prime fasi della ridemocratizzazione. A questo scopo abbiamo visto che, malgrado un sistema di fratture inizialmente condiviso da tutti i paesi, tempi di presentazione, sequenze e intensità delle stesse hanno poi contribuito a diversificare le singole configurazioni partitiche nazionali. Le diverse modalità della transizione accentuano, infine, il quadro delle differenze. Le risposte contenute in questo scritto forniscono solo un primo nucleo parziale di spiegazioni. Tuttavia, nei nostri obiettivi, esso dovrebbe essere un primo passo verso un progetto più ambizioso: la costruzione di un più ampio modello esplicativo delle origini dei diversi sistemi e allineamenti partitici est-europei. 
A ormai cinque anni dal crollo del comunismo, il panorama politico-partitico in Europa orientale appare ancora in fibrillazione. Più in concreto, l'instabilità e la debolezza dei sistemi partitici sono globalmente superiori a quelle che si manifestano nelle neo-democrazie del Sud Europa dagli anni settanta in poi (tab. 4). In queste, infatti, si mette in atto una rotazione al pote$\mathrm{re}^{9}$ senza gravi contraccolpi sui sistemi partitici nel loro complesso: al massimo si hanno singoli casi di partiti in forte declino (per esempio l'UCD spagnola). E il quadro di tale eccezionale instabilità nell'Europa centro-orientale non cambia se il confronto viene allargato all'insieme complessivo dei paesi europei a discontinuità democratica, nei quali la rotazione di partiti al potere, se e quando avviene, non si compie mai subito alle seconde elezioni post-autoritarie $\mathrm{ma}$ in altre successive (Turner 1993, 346).

Si aggiunga poi che l'instabilità partitica nei paesi est-europei non si manifesta solo nella rotazione al governo, ma contempla anche altre trasformazioni clamorose e più concentrate nel tempo che investono il numero e la forza elettorale dei partiti, la frantumazione delle organizzazioni-ombrello, le numerose scissioni e riaggregazioni successive, la comparsa dei partiti nazionalisti. Naturalmente non mancano differenze all'interno del blocco: il sistema partitico ungherese appare più stabile di quelli cecoslovacco (fino a tutto il '92) o polacco; così come i partiti sono più forti e tendono a svolgere maggiormente il ruolo di protagonisti nell'Europa centro-orientale che nei paesi Baltici e in tutti quelli eredi dell'URSS.

A tutt'oggi, infine, lo scenario di instabilità trova continue conferme: basta guardare alle recenti elezioni polacche e ungheresi (settembre '93 e maggio '94) che hanno riportato al potere gli ex comunisti, o al mancato radicamento locale dei partiti (in Polonia, per esempio, circa il $50 \%$ dei sindaci eletti non hanno una chiara affiliazione partitica e dati non molto dissimili sono disponibili anche per l'Ungheria: Elander e Gustafsson 1993, 311; Lomax 1993, 92). Ulteriori manifestazioni di debolezza

9 Mi riferisco all'alternanza tra Centro democratico e socialisti in Spagna, tra Nuova democrazia e socialisti in Grecia e tra socialisti e socialdemocratici in Portogallo. Va però ricordato che tali dinamiche si manifestano in un arco di tempo più lungo dei quattro-cinque anni qui esaminati per l'Europa orientale. 
TAB. 4. Dinamiche dei sistemi partitici in paesi neo-democratici

Italia post-1948

Giappone post-1952

Europa del Sud anni '70 e dopo

Europa orientale post-1989
Effetto freezing delle

prime elezioni democratiche

Rotazioni al potere destra-sinistra (Spagna e Grecia) o viceversa (Portogallo)

Rotazioni al potere, scissioni e frantumazione di partiti, variazione nel numero e nella forza elettorale, variabilità e instabilità dei conflitti e delle issues

partitica sono poi: la persistente personalizzazione della politica (c'è una tendenza diffusa a identificare il partito con il leader principale), l'astensionismo elettorale (forte specialmente in Polonia, Ungheria, oltre che in alcuni stati eredi dell'URSS), un'estesa ostilità contro la stessa nozione di «partito» ${ }^{10}$. Tutto questo mette in luce una contraddizione. Da un lato le transizioni si sviluppano attraverso i partiti: appare del tutto logico che siano essi i protagonisti del cambiamento e non altre istituzioni quali i militari, la polizia, personalità forti e carismatiche, ecc. Dall'altro, però, le formazioni partitiche sono deboli, stentano ad affermarsi e a consolidarsi.

A cosa attribuire il perpetuarsi di un tale quadro di instabilità? Una prima risposta sta nel clima di incertezza circa l'evoluzione della conformazione statale all'interno della quale i partiti agiscono. Tale è certamente il caso della Cecoslovacchia e dei paesi successori dell'URSS e della Jugoslavia. Ma in parte tale clima esiste in tutta la regione, come effetto (e anche causa) della rinascita del nazionalismo, della debolezza statale, della non definitività delle istituzioni centrali e dei confini degli stati. È difficile dire se anche l'incertezza delle varie normative elettorali (in Polonia, per esempio, alle elezioni del 1989, 1991 e 1993 si vota ogni volta con un sistema diverso) possa esser fatta rientrare in questo quadro: certo è che neanche essa concorre alla stabilità del quadro partitico.

Altre cause sono poi la presenza di sistemi di articolazione

10 Che quasi mezzo secolo di partito unico ne sia o no la causa principale è difficile dire. Resta comunque il fatto che in questi paesi sono molti i segni che confermano l'immagine del partito politico come assai screditata. Sotto questo profilo è significativo lo slogan del Foro Civico in Cecoslovacchia nel 1990: «I partiti sono per gli iscritti ai partiti; noi siamo a favore del popolo». 
degli interessi ancora fragili e non ben strutturati e l'assenza di un sistema di fratture forte, stabile e consolidato strutturalmen$\mathrm{te}^{11}$. Sul punto va anche rilevato che i tre tipi di conflitto (innovazione/conservazione, pro/anti-mercato e etno-territoriale) che concorrono a modellare i sistemi partitici est-europei costituiscono in fondo le diverse facce di un'unica grande contrapposizione tra una cultura individualistica e cosmopolita mirante alla valorizzazione del merito e delle capacità personali, ed una invece allo stesso tempo più collettivistica e particolaristica.

Un ultimo aspetto da sottolineare è che i partiti est-europei sono in buona parte di nuova formazione. Con l'eccezione di quelli eredi del partito unico o di eventuali partiti «satelliti» (per esempio in Polonia), mancano infatti di precedenti esperienze organizzative e strutturali, così come mancano quasi del tutto (dopo la morte di Dubček, Walesa è forse la sola eccezione) di leaders forti di una qualche precedente esperienza politica. Anche nel caso dei partiti ex comunisti (e di quelli ex satelliti) non solo c'è stato un rinnovamento più o meno ampio dell'ideologia e dei programmi, ma l'intero corpo dirigente è stato sostituito e l'organizzazione ha subito un forzato ridimensionamento e riadattamento.

Certo, i partiti eredi del vecchio regime mantengono un vantaggio organizzativo rispetto agli altri, ma esso da solo non basta a vincere le elezioni. Infatti, una volta superata una soglia minima indispensabile (al di sotto della quale un partito diventa «invisibile» agli occhi dei più), la variabile organizzativa tende a declinare d'importanza. Gunther, Sani e Shabad $(1986,393)$ ricordano il caso del PC spagnolo nel 1977 che, malgrado la struttura più sviluppata, la leadership locale meglio preparata, il sostegno dei sindacati più importanti e una presenza capillare, non arrivò a prendere un terzo dei voti dei meno organizzati socialisti.

Per concludere, l'Europa centro-orientale si pone globalmente a metà strada tra l'Europa del Sud, i cui sistemi partitici si sono rapidamente assimilati a quelli del resto d'Europa, e l'America latina il cui sviluppo partitico è stato invece condizio-

"Se il sistema di fratture dovesse continuare a mantenersi debole e non strutturato sarebbe prevedibile una continuazione della instabilità elettorale. Infatti, «quanto più elevata e pervasiva è la forza del sistema di fratture di un dato paese o periodo, più bassa sarà l'elasticità del voto e perciò più basso anche il livello di instabilità elettorale» (Bartolini e Mair 1990, 212). 
nato $\mathrm{da}$ aspetti molto peculiari ${ }^{12}$. Più specificamente, il fatto che quella est-europea non sia solo una transizione politica, ma sia altresì una transizione economica $\mathrm{e}$, in molti casi, anche della forma statale dà la misura di alcune particolarità dello sviluppo partitico di questi paesi. Sviluppo che appare dunque condizionato dai conflitti relativi alle riforme economiche (quale e quanto spazio dare al mercato) e da quelli di natura etno-territoriale. E su tali fratture, sulla loro evoluzione e sul loro intrecciarsi, si giocherà probabilmente il consolidamento di queste nuove democrazie.

\section{Riferimenti bibliografici}

Baloyra, E. (a cura di) (1987), Comparing New Democracies, Boulder and London, Westview Press.

Bartolini, S. e P. Mair (1990), Identity, Competition and Electoral Availability. The Stabilisation of European Electorates 1885-1985, Cambridge, Cambridge University Press.

Batt, J. (1991), The End of Communist Rule in East-Central Europe: A Four-Country Comparison, in «Government and Opposition», XXVI, pp. 368-390.

von Beyme, K. (1992), L'europeizzazione dell'Europa orientale, in Calise (1992), pp. 275-297.

Blaha, J. e F. Wehrlé (1992), La Fédération tchèque et slovaque mise en cause: aspects politiques et économiques, in «Le Courier des pays de l'Est», n. 370, pp. 44-56.

Bowers, S.R. (1992), Etbnic Politics in Eastern Europe, in «Conflict Studies», n. 248, febbraio.

Caccamo, D. (1991), Introduzione alla storia dell'Europa orientale, Roma, La Nuova Italia Scientifica.

Calise, M. (a cura di) (1992), Come cambiano i partiti, Bologna, Il Mulino.

Capek, A. e G.W. Sazama (1993), Czech and Slovak Economic Relations, in «Europe-Asia Studies», XLV, pp. 211-235.

Cotta, M. (1992a), Continuità e discontinuità nei sistemi partitici europei, in Calise (1992), pp. 207-241.

- (1992b), New Party Systems after the Dictatorship: Dimensions of Analysis. The East European Cases in Comparative Perspective,

12 Tra i quali l'assenza di alcune delle fratture che hanno caratterizzato il mondo occidentale e lo sradicamento di gran parte dei partiti formatisi prima dell'inizio della politica di massa (Dix 1989, 26). 
Università degli Studi di Siena, Dipartimento di Studi politici e di Storia giuridico-politica, Working paper n. 9.

Deis, M.J. (1992), A Study of Nationalism in Czechoslovakia, in «RFE/ RL Research Report», 31 gennaio, pp. 8-13.

Dix, R.H. (1989), Cleavage Structures and Party Systems in Latin America, in «Comparative Politics», XXII, pp. 23-37.

Dogan, M. (1992), Il declino dei nazionalismi in Europa occidentale, in «Modernizzazione e Sviluppo», 1-2, pp. 5-15.

- (1993), Le nationalisme en Europe: déclin à l'Ouest, résurgence à l'Est, in Philippart (1993), pp. 141-174.

Elander, I. e M. Gustafsson (1993), The Re-emergence of Local Self-government in Central Europe. Some Notes on the First Experience, in «European Journal of Political Research», XXIII, 295-322.

Fisichella, D. (1993), Elezioni e democrazia, Bologna, Il Mulino.

Grilli di Cortona, P. (1989), Le crisi politiche nei regimi comunisti, Milano, Franco Angeli.

- (1991a), Dal comunismo alla democrazia in Europa centrale: Ungheria e Cecoslovacchia, in «Rivista Italiana di Scienza Politica», XXI, pp. 281-313.

- (1991b), Rethinking Regime Change in Hungary and Czechoslovakia, in «International Social Science Journal», n. 128, pp. 315. 330.

- (1994), L'evoluzione dei sistemi di partito nell'Europa centro-orientale, relazione presentata alle «Giornate Amedeo Giannini» su: «La legislazione elettorale degli Stati dell'Europa centro-orientale», Roma, 27-28 gennaio.

Gunther, R., G. Sani e G. Shabad (1986), Spain After Franco. The Making of a Competitive Party System, Berkeley, University of California Press.

Hankiss, E. (1990), East European Alternatives, Oxford, Clarendon Press.

Hibbing, J.R. e S.C. Patterson (1992), A Democratic Legislature in the Making. The Historic Hungarian Elections of 1990, in «Comparative Political Studies», XXIV, pp. 430-454.

Higley, J. e M.G. Burton (1989), The Elite Variable in Democratic Transitions and Breakdowns, in «American Sociological Review», LIV, pp. 17-32.

Huntington, S.P. (1991), The Third Wave. Democratization in the Late Twentieth Century, Norman and London, University of Oklahoma Press.

Jasiewicz, K. (1992), From Solidarity to Fragmentation, in «Journal of Democracy», III, pp. 55-69.

Kitschelt, H. (1992), The Formation of Party Systems in East Central Europe, in «Politics \& Society», XX, pp. 7-50.

Körösényi, A. (1990), Hungary, in «Electoral Studies», IX, pp. 337345. 
Lijphart, J. (1992), Democratization and Constitutional Choices in Czecho-Slovakia, Hungary and Poland, in «Journal of Theoretical Politics», IV, pp. 207-223.

Linz, J.J. e A. Stepan (1992), Political Identities and Electoral Sequences: Spain, the Soviet Union, and Yugoslavia, in «Daedalus», CXXI, pp. 123-139.

Lipset, S.M. e S. Rokkan (1967), Cleavage Structures, Party Systems, and Voter Alignments: An Introduction, in S.M. Lipset e S. Rokkan (a cura di) (1967), Party Systems and Voter Alignments: Cross-National Perspectives, New York, The Free Press, pp. 1-64.

Lomax, B. (1993), Hungary, in Whitefield (1993), pp. 79-98.

McGregor, J. (1993), How Electoral Laws Shape Eastern Europe's Parliaments, in «RFE/RL Research Report», 2, 4, 22 gennaio, pp. 1118.

Morlino, L. (1987), Democratic Establishments: A Dimensional Analysis, in Baloyra (1987), pp. 53-78.

- (1992), Partiti e consolidamento democratico nel Sud Europa, in Calise (1992), pp. 243-274.

O'Donnell, G. e P.C. Schmitter (1986), Convoking Elections (and Provoking Parties), in G. O'Donnell, P. Schmitter e L. Whitehead (1986), pp. 57-64, vol. IV.

O'Donnell, G., P.C. Schmitter e L. Whitehead (a cura di) (1986), Transitions from Autboritarian Rule. Prospects for Democracy, voll. IV, Baltimore e London, The Johns Hopkins University Press.

Offe, C. (1992), Vers le capitalisme par construction démocratique? La théorie démocratique et la triple transition en Europe de l'Est, in «Revue Française de Science Politique», XLII, pp. 923-942.

Papadakis, A. (1988), The Historical Tradition of Church-State Relations under Orthodoxy, in Ramet (1988), pp. 37-58.

Pataki, J. (1991), Popularity of Major Political Parties Decreases, in «Report on Eastern Europe», 2 agosto, pp. 5-10.

Philippart, E. (a cura di) (1993), Nations et frontières dans la nouvelle Europe. L'impact croisé, Bruxelles, Editions Complexe.

Ramet, P. (1988), Autocephaly and National Identity in Church-State Relations in Eastern Christianity: An Introduction, in P. Ramet (a cura di), Eastern Christianity and Politics in the Twentieth Centu$r y$, Durham e London, Duke University Press.

Rokkan, S. (1982), Cittadini, elezioni, partiti, Bologna, Il Mulino.

Sartori, G. (1976), Parties and Party Systems, New York, Cambridge University Press.

Shain, Y. e J.J. Linz (1992), The Role of Interim Governments, in «Journal of Democracy», III, pp. 73-89.

Tarrow, S. (1991), «Aiming at a Moving Target»: Social Science and the Recent Rebellions in Eastern Europe, in «PS: Political Science and Politics», XXIV, pp. 12-20. 
Tilly, C. (1993), European Revolutions 1492-1992, Oxford, Basil Blackwell; trad. it. Le rivoluzioni europee 1492-1992, Roma-Bari, Laterza, 1993.

Turner, A.W. (1993), Postauthoritarian Elections. Testing Expectations About «First» Elections, in «Comparative Political Studies», XXVI, pp. 330-349.

Wawrzyniak, J. (1994), La legislazione elettorale in Polonia, relazione presentata alle «Giornate Amedeo Giannini» su: «La legislazione elettorale degli Stati dell'Europa centro-orientale», Roma, 27-28 gennaio.

Webb, W.L. (1992), The Polish General Election of 1991, in «Electoral Studies», XI, pp. 166-170.

White, S. (1990), The Elections to the USSR Congress of People's Deputies March 1989, in «Electoral Studies», IX, pp. 59-66.

Whitefield, S. (a cura di) (1993), The New Institutional Architecture of Eastern Europe, New York, St. Martin's Press.

Wightman, G. (1993), The Czechoslovak Parliamentary Elections of 1992 , in «Electoral Studies», XII, pp. 83-86. 